\title{
MATHEMATICAL JUSTIFICATION OF THE HYDROSTATIC APPROXIMATION IN THE PRIMITIVE EQUATIONS OF GEOPHYSICAL FLUID DYNAMICS*
}

\author{
PASCAL AZÉRAD ${ }^{\dagger}$ AND FRANCISCO GUILLÉN ${ }^{\ddagger}$
}

\begin{abstract}
Geophysical fluids all exhibit a common feature: their aspect ratio (depth to horizontal width) is very small. This leads to an asymptotic model widely used in meteorology, oceanography, and limnology, namely the hydrostatic approximation of the time-dependent incompressible Navier-Stokes equations. It relies on the hypothesis that pressure increases linearly in the vertical direction. In the following, we prove a convergence and existence theorem for this model by means of anisotropic estimates and a new time-compactness criterium.
\end{abstract}

Key words. Navier-Stokes equations, shallow domains, geophysical fluid dynamics, hydrostatic approximation, singular perturbation, compactness criterium, asymptotic analysis

AMS subject classifications. 35Q30, 35B40, 76D05, 34C35

PII. S0036141000375962

1. Introduction. Atmospheric flow in meteorology, water flow in oceanography, and limnology are all described by the Navier-Stokes equations. Due to the fact that the aspect ratio

$$
\epsilon=\frac{\text { characteristic depth }}{\text { characteristic width }}
$$

is very small in most geophysical domains, asymptotic models have been used; see, e.g., $[9,15,22]$. One such model is the primitive equations model; see, e.g., [11, 12], wherein the unknown flow variables are velocity, pressure, temperature, and salinity (in the case of an ocean). Besides, most geophysical fluids are stratified (i.e., density is a known function of the temperature (and salinity, if any)) and have a free surface. We shall not investigate these features in this paper, leaving it, rather, for forthcoming work.

Instead we shall focus on the assumption that the pressure is hydrostatic, i.e., increases linearly with respect to the depth, as in the static case. This law agrees well with experiment (as first observed by Blaise Pascal around 1650; see [14])) and is frequently taken as a hypothesis in geophysical fluid dynamics. We justify this assumption by means of asymptotic analysis (taking $\epsilon$ as the small parameter). Our derivation is made possible by the use of anisotropic eddy viscosities, namely $\nu=$ $\left(\nu_{x}, \nu_{y}, \nu_{z}\right)$, relying on the fact that the ratio between the horizontal and vertical scales leads to very different sizes for the horizontal and vertical eddies (see $[9,15])$. Specifically, if we assume that $\nu=\left(\nu_{1}, \nu_{2}, \epsilon^{2} \nu_{3}\right)$ with $\nu_{i}=\mathrm{O}(1)$ for $i=1,2,3$, then we will see that weak solutions of the Navier-Stokes equations converge to a weak solution of a limit problem with hydrostatic pressure.

* Received by the editors July 26, 2000; accepted for publication (in revised form) August 22, 2001; published electronically December 18, 2001. This work was supported by the fonds Franco-Espagnol D.R.E.I.F. (ref. UC 815) and the project MAR98-0486 C.I.C.Y.T. (España).

http://www.siam.org/journals/sima/33-4/37596.html

${ }^{\dagger}$ Laboratoire de Modélisation, Analyse Non Linéaire et Optimisation, Université de Perpignan, 52 av. de Villeneuve, F-66860 Perpignan cedex, France (azerad@univ-perp.fr).

‡Departamento de Ecuaciones Diferenciales y Análisis Numérico, c/ tarfia s/n Universidad de Sevilla, 41012 Sevilla, Spain (guillen@numer.us.es). 
The stationary case has already been studied (see [4] for the linear problem and [5] for the nonlinear one), whereas the linear time-dependent case was solved in [1]. The main task of this paper is then to solve the nonlinear time-dependent case. Our result was announced in [2], whereas numerical simulations stemming from it were discussed in [3].

Fluid flow in thin domains (flat, curved, and with various boundary conditions) has been extensively studied; see [7, 13, 16, 20, 21]. In these works, an isotropic viscosity is used, and the depth is constant. By averaging along the vertical direction, two-dimensional (2D) limit models are obtained, together with existence and global regularity results.

Our approach is different, because we neither eliminate the vertical velocity by averaging nor assume the depth of the domain to be constant. By making use of different horizontal and vertical eddy viscosities, we are able to derive a three-dimensional (3D) limit nonlinear model. Let us emphasize that the anisotropic viscosity hypothesis is fundamental for the derivation of the primitive equations: in the stationary case, keeping an isotropic viscosity, the asymptotic model is linear, with vanishing horizontal diffusion; see [6].

The paper is organized as follows. In section 2, we present the physical model and the scaling leading to the primitive equations. We state the main theorem in section 3. The functional setting and weak formulation are described in section 4 . In the next section, we state and prove a time-compactness result, which we shall use in the proof of the main theorem in section 6 . Finally, in section 7 , we comment on the convergence of the pressure and the orders of magnitude of the vertical velocity with respect to the aspect ratio.

2. Equations governing the flow and scaling. Let us consider an incompressible homogeneous fluid filling a thin domain defined by

$$
\Omega_{\epsilon}=\left\{(x, y, z) \in \mathbb{R}^{3} ;(x, y) \in \omega,-\epsilon h(x, y)<z<0\right\},
$$

where $\omega$ is an open bounded Lipschitz domain in $\mathbb{R}^{2}$ and $h: \bar{\omega} \rightarrow \mathbb{R}$ is a nonnegative lipschitzian application, which is arbitrary provided that $\Omega_{\epsilon}$ is lipschitzian. In particular, $h$ may vanish, contrary to $[12,9]$, but in order that the domain $\Omega_{\epsilon}$ has no cusps, the slope must not vanish on the shores. ${ }^{1}$ We denote by $\Gamma_{s}=\bar{\omega} \times\{0\}$ the fluid surface and by $\Gamma_{b}^{\epsilon}=\partial \Omega_{\epsilon} \backslash \Gamma_{s}$ the basin bottom. The fluid flow in $\Omega_{\epsilon}$ is generated by the wind traction on the surface $\Gamma_{s}$, influenced by the Coriolis and centrifugal forces and governed by the Navier-Stokes equations, in which we take different eddy viscosities according to the direction; see $[5,9,15]$. Finally, we take the density as identically equal to one. In a geophysical rotating frame ( $z$ pointing upwards, $x$ east, and $y$ north), the initial-boundary value problem reads as follows.

Find $\mathbf{v}=\left(v_{1}, v_{2}, v_{3}\right)$ (velocity) and $q$ (pressure), such that

$$
\begin{array}{rlrl}
\partial_{t} \mathbf{v}+(\mathbf{v} \cdot \nabla) \mathbf{v}-\Delta_{\nu} \mathbf{v}+\nabla q+2 \mathbf{w} \times \mathbf{v}=\mathbf{g} & & \text { in } \Omega_{\epsilon} \times(0, \mathrm{~T}), \\
\operatorname{div} \mathbf{v}=0 & & \text { in } \Omega_{\epsilon} \times(0, T), \\
\mathbf{v}=0 & & \text { on } \Gamma_{b}^{\epsilon} \times(0, T), \\
\nu_{z} \partial_{z} v_{1}=\tau_{1}, & \nu_{z} \partial_{z} v_{2}=\tau_{2}, \quad v_{3}=0 & & \text { on } \Gamma_{s} \times(0, T), \\
\mathbf{v}(\cdot, t=0) & =\mathbf{v}_{0} & & \text { in } \Omega_{\epsilon} .
\end{array}
$$

\footnotetext{
${ }^{1}$ This is a technical hypothesis. One could probably dispense with it due to the specific shape of the domain.
} 
In (2.1), $\nabla=\left(\partial_{x}, \partial_{y}, \partial_{z}\right)$ denotes the gradient vector, and $\Delta_{\nu}$ denotes the anisotropic Laplacian defined by $\Delta_{\nu}=\nu_{x} \partial_{x x}^{2}+\nu_{y} \partial_{y y}^{2}+\nu_{z} \partial_{z z}^{2}$ with $\nu=\left(\nu_{x}, \nu_{y}, \nu_{z}\right)$ the eddy kinematic viscosity vector. Moreover, $\mathbf{w}=f(0, \cos (l(y)), \sin (l(y)))$ represents the earth rotation angular speed ( $f$ the module and $l(y)$ the latitude), $2 \mathbf{w} \times \mathbf{v}$ represents the Coriolis acceleration $\left(x\right.$ denotes the cross-product in $\left.\mathbb{R}^{3}\right)$, and $\mathbf{g}$ represents the force due to gravity (which also includes the centrifugal effect). It is well known (cf. [15, p. 18]) that $\mathbf{g}$ is a potential, i.e., $\mathbf{g}=\nabla \varphi$. It is customary to incorporate the gravity potential in the pressure term; thus we set

$$
p=q-\varphi
$$

Equation (2.2) represents the incompressibility condition, and (2.3) represents the no-slip condition on the bottom.

In (2.4), $\tau_{i}, i=1,2$, stand for the horizontal tractions exerted by the wind on the (fixed) surface $\Gamma_{s}$ of the fluid, and $w=0$ on $\Gamma_{s}$ comes from the rigid lid hypothesis. In $(2.5), \mathbf{v}_{0}=\left(v_{01}, v_{02}, v_{03}\right)$ designates the initial velocity.

Remark. We have neglected the earth's curvature, and hence our analysis is valid only locally, e.g., for lakes; for seas or oceans, spherical coordinates should be used [12], although this can be somewhat cumbersome.

As usual in asymptotic analysis, we perform a vertical scaling to make the domain independent of $\epsilon$, that is,

$$
x=x_{1}, \quad y=x_{2}, \quad z=\epsilon x_{3},
$$

so that $\Omega=\left\{\left(x_{1}, x_{2}, x_{3}\right) \in \mathbb{R}^{3} ;\left(x_{1}, x_{2}\right) \in \omega,-h\left(x_{1}, x_{2}\right)<x_{3}<0\right\}$ is the new fixed domain.

The corresponding kinematic scaling is

$$
v_{1}=u_{1}^{\epsilon}, \quad v_{2}=u_{2}^{\epsilon}, \quad v_{3}=\epsilon u_{3}^{\epsilon}, \quad p=p^{\epsilon},
$$

so that $\mathbf{u}^{\epsilon}=\left(u_{1}^{\epsilon}, u_{2}^{\epsilon}, u_{3}^{\epsilon}\right)$ is the new unknown velocity and $p^{\epsilon}$ is the new pressure.

It is necessary to scale the mechanical quantities accordingly. First, it is only natural to assume $v_{01}=u_{01}, v_{02}=u_{02}$, and $v_{03}=\epsilon u_{03}$, where $u_{0 i}$ does not depend on $\epsilon, i=1,2,3$. Next we assume $\nu_{x}=\nu_{1}, \nu_{y}=\nu_{2}$, and $\nu_{z}=\epsilon^{2} \cdot \nu_{3}$, where $\nu_{1}, \nu_{2}, \nu_{3}$ are constants. As mentioned in the introduction, in oceanography the vertical eddy viscosity is usually very small compared to the horizontal one. We refer to [5] for a mathematical discussion of this assumption, and here we content ourselves with one heuristic comment. Basically, a kinematic viscosity has the dimension $L^{2} / T$, where $L$ (resp., $T$ ) is a typical length (resp., time) scale so that $\nu_{x}$ and $\nu_{y}$ have the dimension $L_{H}^{2} / T$, whereas $\nu_{z}$ has the dimension $L_{V}^{2} / T$, where $L_{H}$ (resp., $L_{V}$ ) denotes a typical horizontal (resp., vertical) length scale. It follows that the ratio $\nu_{z} / \nu_{x}$ and $\nu_{z} / \nu_{y}=\mathrm{O}\left(\epsilon^{2}\right) .^{2}$

Now (2.4) becomes

$$
\nu_{3} \partial_{3} u_{i}^{\epsilon}=\tau_{i}^{\epsilon} / \epsilon, \quad i=1,2 .
$$

We see that in order to end up with an $\mathrm{O}(1)$-wind force on the rescaled domain, we have to assume that $\tau_{i}^{\epsilon}=\epsilon \cdot \theta_{i}, i=1,2$, where the $\theta_{i}$ are functions independent of $\epsilon$.

\footnotetext{
${ }^{2}$ We do not delude ourselves with this sketchy argument. As far as we know, up to now there has been no rigorous derivation of any eddy viscosity model.
} 
Remark. This last assumption can also be motivated by dimensional analysis, as follows. From $\tau_{i}=\nu_{z} \partial_{z} v_{i}$, one derives that $\tau_{i}$ has the dimension of

$$
\frac{L_{V}^{2}}{T} \cdot \frac{1}{L_{V}} \cdot \frac{L_{H}}{T}=\epsilon \frac{L_{H}^{2}}{T^{2}}=O(\epsilon)
$$

With the above considerations, problem (2.1)-(2.5) transforms into the following anisotropic Navier-Stokes equations:

$$
\begin{aligned}
& \partial_{t} u_{1}^{\epsilon}+\mathbf{u}^{\epsilon} \cdot \nabla u_{1}^{\epsilon}-\Delta_{\nu} u_{1}^{\epsilon}-\alpha u_{2}^{\epsilon}+\epsilon \beta u_{3}^{\epsilon}+\partial_{1} p^{\epsilon}=0 \quad \text { in } \Omega \times(0, T), \\
& \partial_{t} u_{2}^{\epsilon}+\mathbf{u}^{\epsilon} \cdot \nabla u_{2}^{\epsilon}-\Delta_{\nu} u_{2}^{\epsilon}+\alpha u_{1}^{\epsilon}+\partial_{2} p^{\epsilon}=0 \quad \text { in } \Omega \times(0, T), \\
& \epsilon^{2}\left\{\partial_{t} u_{3}^{\epsilon}+\mathbf{u}^{\epsilon} \cdot \nabla u_{3}^{\epsilon}-\Delta_{\nu} u_{3}^{\epsilon}\right\}-\epsilon \beta u_{1}^{\epsilon}+\partial_{3} p^{\epsilon}=0 \quad \text { in } \Omega \times(0, T) \text {, } \\
& \operatorname{div} \mathbf{u}^{\epsilon}=0 \text { in } \Omega \times(0, T), \\
& \mathbf{u}^{\epsilon}=0 \text { on } \Gamma_{b} \times(0, T), \\
& \nu_{3} \partial_{3} u_{1}^{\epsilon}=\theta_{1}, \quad \nu_{3} \partial_{3} u_{2}^{\epsilon}=\theta_{2}, \quad u_{3}^{\epsilon}=0 \quad \text { on } \Gamma_{s} \times(0, T), \\
& \mathbf{u}^{\epsilon}(\cdot, t=0)=\mathbf{u}_{0} \quad \text { in } \Omega .
\end{aligned}
$$

Now $\nabla=\left(\partial_{1}, \partial_{2}, \partial_{3}\right), \Delta_{\nu}=\nu_{1} \partial_{11}^{2}+\nu_{2} \partial_{22}^{2}+\nu_{3} \partial_{33}^{2}, \Gamma_{b}=\partial \Omega \backslash \Gamma_{s}, \alpha=2 f \sin \left(l\left(x_{2}\right)\right)$, and $\beta=2 f \cos \left(l\left(x_{2}\right)\right)$.

If we assume that $\mathbf{u}^{\epsilon}=O(1)$, then neglecting the $\epsilon^{2}$ and $\epsilon$ terms in the first and third momentum equation, (2.7) and (2.9), we formally get the hydrostatic NavierStokes equations, also called the primitive equations:

$$
\begin{aligned}
& \partial_{t} u_{1}+\mathbf{u} \cdot \nabla u_{1}-\Delta_{\nu} u_{1}-\alpha u_{2}+\partial_{1} p=0 \quad \text { in } \Omega \times(0, \mathrm{~T}), \\
& \partial_{t} u_{2}+\mathbf{u} \cdot \nabla u_{2}-\Delta_{\nu} u_{2}+\alpha u_{1}+\partial_{2} p=0 \quad \text { in } \Omega \times(0, \mathrm{~T}), \\
& \partial_{3} p=0 \text { in } \Omega \times(0, \mathrm{~T}), \\
& \operatorname{div} \mathbf{u}=0 \quad \text { in } \Omega \times(0, T), \\
& u_{1}=u_{2}=u_{3} n_{3}=0 \quad \text { on } \Gamma_{b} \times(0, T), \\
& \nu_{3} \partial u_{1}=\theta_{1}, \quad \nu_{3} \partial_{3} u_{2}=\theta_{2}, \quad u_{3}=0 \quad \text { on } \Gamma_{s} \times(0, T), \\
& u_{i}(\cdot, t=0)=u_{0 i} \quad \text { in } \Omega, i=1,2 .
\end{aligned}
$$

Remark. The boundary condition (2.18) differs from its counterpart (2.11) because $u_{3}$ is less regular than $u_{1}, u_{2}$ as we shall see below. Also, the initial condition (2.20) does not involve $u_{3}$, the time derivative of which is missing in the hydrostatic model. The problem is not in the Cauchy-Kowalevska form. ${ }^{3}$

Remark. If $u_{3}$ were to be computed directly from (2.17), which is a first order equation, it is not obvious at all that it would fulfill the two boundary conditions on the bottom (2.18) and the surface (2.19).

3. Main theorem. Let $T$ be a fixed positive duration. We make the natural assumption of a wind of finite energy: $\theta_{1}, \theta_{2} \in L^{2}\left(0, T ; H^{-1 / 2}\left(\Gamma_{s}\right)\right)$. Our main result is the following theorem.

Theorem 3.1. Let $\mathbf{u}_{0} \in L^{2}(\Omega)^{3}$, with $\operatorname{div} \mathbf{u}_{0}=0, \mathbf{u}_{0} \cdot \mathbf{n}=0$ on $\partial \Omega$, and $\theta_{1}, \theta_{2} \in$ $L^{2}\left(0, T ; H^{-1 / 2}\left(\Gamma_{s}\right)\right)$; there exists a weak solution $\mathbf{u}$ of the hydrostatic Navier-Stokes equations (2.14)-(2.20), obtained as a limit of weak solutions $\mathbf{u}^{\epsilon}$ of the anisotropic Navier-Stokes equations (2.7)-(2.13), as the aspect ratio $\epsilon$ tends to zero.

\footnotetext{
${ }^{3}$ Meteorologists say that $u_{3}$ is no longer a prognostic variable (see $[11,12]$ ).
} 
The proof relies on a priori estimates in anisotropic spaces (Propositions 6.1 and 6.2), which are sufficient to take the limit in the linear terms (see [1]), whereas for the nonlinear terms, we establish a new time-compactness criterium (Theorem 5.1), which enables us to get strong convergence of the horizontal velocities; see Lemma 6.3. This theorem states essentially that a small perturbation of an $L^{p}$-equicontinuous family still possesses a strong convergent subsequence. Let us emphasize that this seemingly technical refinement is by no means superfluous. Indeed, the usual compactness estimate fails: as $\left(u_{1}^{\epsilon}, u_{2}^{\epsilon}, \epsilon^{2} u_{3}^{\epsilon}\right)$ is not divergence free, even if it is easy from (2.7)-(2.9) to control $\partial_{t}\left(u_{1}^{\epsilon}, u_{2}^{\epsilon}, \epsilon^{2} u_{3}^{\epsilon}\right)$ in some dual space of divergence free velocities, it is not possible to apply the Aubin-Lions lemma to get compactness.

Another major difficulty of the proof is the lack of regularity of the vertical velocity, which is determined only by the incompressibility equation (2.10).

Remark. It is possible to handle a general force $\left(f_{1}, f_{2}, f_{3}\right)$ in problem (2.14)(2.20), by simply adding $\mathbf{f}=\left(f_{1}, f_{2}, \frac{f_{3}}{\epsilon}\right)$ to $(2.1)$, in order to end up with $\left(f_{1}, f_{2}, f_{3}\right)$ in $(2.7)-(2.9)$.

4. Weak formulation and anisotropic spaces. We need the following Hilbert spaces:

$$
H_{b}^{1}(\Omega)={\overline{C_{b}^{\infty}(\Omega)}}^{H^{1}(\Omega)}=\left\{v \in H^{1}(\Omega) ; v=0 \text { on } \Gamma_{b}\right\}
$$

(where $C_{b}^{\infty}(\Omega)=\left\{\varphi \in C^{\infty}(\bar{\Omega}) ; \varphi=0\right.$ in some neighborhood of $\left.\Gamma_{b}\right\}$ ),

$$
\begin{gathered}
\mathbf{V}=\left\{\mathbf{v} \in H_{b}^{1}(\Omega) \times H_{b}^{1}(\Omega) \times H_{0}^{1}(\Omega) ; \operatorname{div} \mathbf{v}=0 \text { in } \Omega\right\}, \\
H\left(\partial_{3}, \Omega\right)=\left\{v \in L^{2}(\Omega) ; \partial_{3} v \in L^{2}(\Omega)\right\}
\end{gathered}
$$

(endowed with the norm $\|v\|_{H\left(\partial_{3}, \Omega\right)}^{2}=\|v\|_{L^{2}(\Omega)}^{2}+\left\|\partial_{3} v\right\|_{L^{2}(\Omega)}^{2}$ ),

$$
H_{0}\left(\partial_{3}, \Omega\right)={\overline{C_{0}^{\infty}(\Omega)}}^{H\left(\partial_{3}, \Omega\right)}=\left\{v \in H\left(\partial_{3}, \Omega\right) ; v n_{3}=0 \text { on } \partial \Omega\right\}
$$

( $n_{3}$ is the third component of the normal exterior vector on $\partial \Omega$, and $v n_{3}$ is understood in the $H^{-1 / 2}(\partial \Omega)$ sense (see [19] for these spaces)),

$$
\mathbf{W}=\left\{\mathbf{u} \in H_{b}^{1}(\Omega) \times H_{b}^{1}(\Omega) \times H_{0}\left(\partial_{3}, \Omega\right) ; \operatorname{div} \mathbf{u}=0 \text { in } \Omega\right\} .
$$

Let us denote that $u_{H}=\left(u_{1}, u_{2}\right), \theta_{H}=\left(\theta_{1}, \theta_{2}\right), b\left(u_{H}\right)=\alpha\left(-u_{2}, u_{1}\right)$, and $\nabla_{\nu}=$ $\left(\nu_{1}^{1 / 2} \partial_{1}, \nu_{2}^{1 / 2} \partial_{2}, \nu_{3}^{1 / 2} \partial_{3}\right)$. The scalar product in $L^{2}(\Omega)^{d}$, or the duality $L^{p}(\Omega), L^{p^{\prime}}(\Omega)$, is denoted by $(\cdot, \cdot)$, and the duality $H^{-1 / 2}\left(\Gamma_{s}\right) H^{1 / 2}\left(\Gamma_{s}\right)$, is denoted by $\langle\cdot, \cdot\rangle_{\Gamma_{s}}$.

The weak form of the hydrostatic Navier-Stokes equations (2.14)-(2.20) is then as follows.

Find $\mathbf{u}=\left(u_{H}, u_{3}\right) \in L^{2}(0, T ; \mathbf{W})$, with $u_{H} \in L^{\infty}\left(0, T ; L^{2}(\Omega)^{2}\right)$, such that

$$
\begin{aligned}
& \int_{0}^{T}-\left(u_{H}, \partial_{t} v_{H}\right)-\left(u_{H},(\mathbf{u} \cdot \nabla) v_{H}\right)+\left(b\left(u_{H}\right), v_{H}\right)+\left(\nabla_{\nu} u_{H}, \nabla_{\nu} v_{H}\right) \\
& \quad=-\left(u_{0 H}, v_{H}(0)\right)+\int_{0}^{T}\left\langle\theta_{H}, v_{H}\right\rangle_{\Gamma_{s}}
\end{aligned}
$$

for all $\mathbf{v}=\left(v_{H}, v_{3}\right) \in H^{1}(0, T ; \mathbf{W})$, with $v_{H}(T)=0$ and $\partial_{3} v_{H} \in L^{\infty}\left(0, T ; L^{3}(\Omega)^{2}\right)$. 
Remark. Notice that a weak solution of the Navier-Stokes equations verifies the following regularity:

$$
\mathbf{u} \in L^{2}(0, T ; \mathbf{V}) \cap L^{\infty}\left(0, T ; L^{2}(\Omega)^{3}\right)
$$

(cf. $[8,10,18]$ ). Now the lack of regularity of $u_{3}$ makes it necessary to change $\mathbf{V}$ to W. Moreover, in general, $u_{3} \notin L^{\infty}\left(0, T ; L^{2}(\Omega)\right)$.

Remark. The regularity $L^{\infty}\left(0, T ; L^{3}(\Omega)^{2}\right)$ is required for $\partial_{3} v_{H}$ to give a meaning to $\int_{0}^{T}\left(u_{H}, u_{3} \partial_{3} v_{H}\right) \mathrm{dt}$. The regularity $L^{2}\left(0, T ; L^{\infty}(\Omega)^{2}\right)$ or any interpolated one $L^{2 / a}\left(0, T ; L^{3 /(1-a)}(\Omega)^{2}\right)$ with $0 \leq a \leq 1$ can also be considered.

5. Compactness by perturbation. We give a compactness criterium, new to our knowledge, which generalizes the well-known translation criterium of RieszFréchet-Kolmogorov, extended to the vectorial case by Simon [17]. In the following, $\tau_{h} f(t)$ denotes $f(t+h)$.

THEOREm 5.1. Let $T>0$, and let the Banach spaces $\mathbf{X} \stackrel{\text { compact }}{\hookrightarrow} \mathbf{B} \hookrightarrow \mathbf{Y}$. Let $\left(f_{\epsilon}\right)_{\epsilon>0}$ be a family of functions of $L^{p}(0, T ; \mathbf{X}), 1 \leq p \leq \infty$, with the extra condition $\left(f_{\epsilon}\right)_{\epsilon>0} \subset \mathcal{C}(0, T ; \mathbf{Y})$ if $p=\infty$, such that

(H1) $\left(f_{\epsilon}\right)_{\epsilon>0}$ is bounded in $L^{p}(0, T ; \mathbf{X})$,

(H2) $\left\|\tau_{h} f_{\epsilon}-f_{\epsilon}\right\|_{L^{p}(0, T-h ; \mathbf{Y})} \leq \varphi(h)+\psi(\epsilon)$ with

$$
\left\{\begin{array}{l}
\lim _{h \rightarrow 0} \varphi(h)=0 \\
\lim _{\epsilon \rightarrow 0} \psi(\epsilon)=0
\end{array}\right.
$$

Then the family $\left(f_{\epsilon}\right)_{\epsilon>0}$ possesses a cluster point in $L^{p}(0, T ; \mathbf{B})$ and also in $\mathcal{C}(0, T ; \mathbf{B})$ if $p=\infty$, as $\epsilon \rightarrow 0$.

Proof. It is enough to prove that, for every sequence $\left(\epsilon_{n}\right)_{n}$ such as $\epsilon_{n}>0$ and $\epsilon_{n} \rightarrow 0$, the family $\left(f_{\epsilon_{n}}\right)_{n}$ is relatively compact in $L^{p}(0, T ; \mathbf{B})$. We apply Theorem 5 of Simon [17, p. 84] to the sequence $\left(f_{\epsilon_{n}}\right)_{n}$, while observing that hypothesis (H2) implies that

$$
\left\|\tau_{h} f_{\epsilon_{n}}-f_{\epsilon_{n}}\right\|_{L^{p}(0, T-h ; \mathbf{Y})} \rightarrow 0 \quad \text { as } h \rightarrow 0
$$

uniformly with respect to $n$. Indeed, (H2) implies that

$$
\forall n,\left\|\tau_{h} f_{\epsilon_{n}}-f_{\epsilon_{n}}\right\|_{L^{p}(0, T-h ; \mathbf{Y})} \leq \varphi(h)+\psi\left(\epsilon_{n}\right) .
$$

Let $\epsilon>0$ and then $\exists N$, such that for all $n \geq N, \psi\left(\epsilon_{n}\right) \leq \epsilon / 2$. On the other hand, $\exists \delta>0$, such that for all $h: 0 \leq h<\delta, \varphi(h) \leq \epsilon / 2$. Therefore, we get the estimate

$$
\forall n \geq N \text { and } \forall h: 0 \leq h<\delta, \quad\left\|\tau_{h} f_{\epsilon_{n}}-f_{\epsilon_{n}}\right\|_{L^{p}(0, T-h ; \mathbf{Y})} \leq \epsilon .
$$

In addition, for each $k \leq N, \exists \delta_{k}>0$, such that for all $h: 0 \leq h<\delta_{k}$

$$
\left\|\tau_{h} f_{\epsilon_{k}}-f_{\epsilon_{k}}\right\|_{L^{p}(0, T-h ; \mathbf{Y})} \leq \epsilon .
$$

This follows from the $L^{p}$-continuity by translation of an $L^{p}$ function for $p<\infty$ and for $p=\infty$; this is precisely a hypothesis.

Defining $\eta=\min \left\{\delta, \delta_{1}, \ldots, \delta_{N}\right\}$, we obtain the desired uniform estimate

$$
\forall h: 0 \leq h<\eta, \quad\left\|\tau_{h} f_{\epsilon_{n}}-f_{\epsilon_{n}}\right\|_{L^{p}(0, T-h ; \mathbf{Y})} \leq \epsilon \quad \forall n .
$$

The family $\left(f_{\epsilon_{n}}\right)_{n}$ fulfills the hypotheses of Simon's theorem. 
6. Proof of the main theorem. For simplicity in the notation, from now on, unless we specify otherwise, we will denote $\mathbf{u}=\mathbf{u}^{\epsilon}$ as a weak solution of the anisotropic Navier-Stokes equations (2.7)-(2.13).

6.1. Energy estimates. The usual energy inequality (cf. [10]) for the NavierStokes equations gives, for a.e. $t \in[0, T]$,

$$
\begin{aligned}
& \left\|u_{H}(t)\right\|_{L^{2}}^{2}+\epsilon^{2}\left\|u_{3}(t)\right\|_{L^{2}}^{2}+\int_{0}^{t}\left\{\left\|\nabla_{\nu} u_{H}(\tau)\right\|_{L^{2}}^{2}+\epsilon^{2}\left\|\nabla_{\nu} u_{3}(\tau)\right\|_{L^{2}}^{2}\right\} d \tau \\
& \leq\left\|u_{0 H}\right\|_{L^{2}}^{2}+\epsilon^{2}\left\|u_{03}\right\|_{L^{2}}^{2}+\int_{0}^{t}\left\langle\theta_{H}, u_{H}\right\rangle_{\Gamma_{s}} .
\end{aligned}
$$

Hence we obtain as in the isotropic Navier-Stokes system (cf. [1]) the following proposition.

Proposition 6.1. The sequences $u_{1}, u_{2}, \epsilon u_{3}$ are bounded in $L^{\infty}\left(0, T ; L^{2}(\Omega)\right) \cap$ $L^{2}\left(0, T ; H^{1}(\Omega)\right)$.

For the vertical velocities, we prove the following.

Proposition 6.2. The sequences $u_{3}$ and $\partial_{3} u_{3}$ are bounded in $L^{2}\left(0, T ; L^{2}(\Omega)\right)$; i.e., $u_{3}$ is bounded in $L^{2}\left(0, T ; H_{0}\left(\partial_{3}, \Omega\right)\right)$.

Proof. As $\operatorname{div} \mathbf{u}=0, \partial_{3} u_{3}=-\partial_{1} u_{1}-\partial_{2} u_{2}$ is bounded in $L^{2}\left(0, T ; L^{2}(\Omega)\right)$. Moreover, the Poincaré inequality in the vertical direction, owing to $u_{3}=0$ on $\Gamma_{s}$, yields

$$
\left\|u_{3}\right\|_{L^{2}} \leq h_{\max }\left\|\partial_{3} u_{3}\right\|_{L^{2}}, \quad \text { where } h_{\max }=\max _{\bar{\omega}} h .
$$

Therefore, we have proved the proposition.

6.2. Fractional time derivatives in horizontal spaces. First, we define the auxiliary Hilbert spaces

$$
B_{H}={\overline{P_{H} \mathcal{U}}}^{\left(L^{2}\right)^{2}}, \quad W_{H}={\overline{P_{H} \mathcal{U}}}^{\left(H^{1}\right)^{2}}, \quad \text { and } \quad Y_{H}={\overline{P_{H} \mathcal{U}}}^{\left(H^{2}\right)^{2}},
$$

where

$$
\mathcal{U}=\left\{\varphi \in C_{b}^{\infty}(\Omega)^{2} \times C_{0}^{\infty}(\Omega) ; \operatorname{div} \varphi=0\right\}
$$

and $P_{H}$ is the projection

$$
P_{H}:\left(x_{1}, x_{2}, x_{3}\right) \in \mathbb{R}^{3} \mapsto\left(x_{1}, x_{2}\right) \in \mathbb{R}^{2} .
$$

Then, from the Sobolev-Rellich embeddings, one deduces easily that

$$
Y_{H} \hookrightarrow W_{H} \hookrightarrow B_{H} \equiv B_{H}^{\prime} \hookrightarrow W_{H}^{\prime} \hookrightarrow Y_{H}^{\prime},
$$

where all are dense and compact embeddings. Here and henceforth, $X^{\prime}$ denotes the dual space of $X$.

Now, we have the following lemma.

LEMma 6.3. The estimate $\left\|\tau_{h} u_{H}-u_{H}\right\|_{L^{\infty}\left(0, T-h ; Y_{H}^{\prime}\right)} \leq C\left(h^{1 / 4}+\epsilon\right)$ holds.

Proof. The spatial weak form of the Navier-Stokes equation (2.7)-(2.13) is

$$
\begin{aligned}
& \frac{d}{d t}\left(u_{H}, v_{H}\right)-\left(u_{H},(\mathbf{u} \cdot \nabla) v_{H}\right)+\left(b\left(u_{H}\right), v_{H}\right)+\left(\nabla_{\nu} u_{H}, \nabla_{\nu} v_{H}\right) \\
& \quad+\epsilon^{2}\left\{\frac{d}{d t}\left(u_{3}, v_{3}\right)+\left(\mathbf{u} \cdot \nabla u_{3}, v_{3}\right)+\left(\nabla_{\nu} u_{3}, \nabla_{\nu} v_{3}\right)\right\} \\
& \quad+\epsilon\left\{\left(\beta u_{3}, v_{1}\right)-\left(\beta u_{1}, v_{3}\right)\right\}=\left\langle\theta_{H}, v_{H}\right\rangle_{\Gamma_{s}} \quad \text { in } \mathcal{D}^{\prime}(0, T) \\
& \quad \forall \mathbf{v}=\left(v_{H}, v_{3}\right) \in \mathbf{V}
\end{aligned}
$$


Letting $v_{H} \in Y_{H}$, there is a null divergence lifting $\mathbf{v}=\left(v_{H}, v_{3}\right) \in H_{b}^{2}(\Omega)^{2} \times H_{0}^{1}\left(\partial_{3}, \Omega\right)$ such that

$$
\left\|v_{3}\right\|_{H^{1}}+\left\|\partial_{3} v_{3}\right\|_{H^{1}} \leq C\left\|v_{H}\right\|_{Y_{H}} .
$$

Here, the spaces $H_{b}^{2}(\Omega)$ and $H_{0}^{1}\left(\partial_{3}, \Omega\right)$ are the natural extensions of the spaces $H_{b}^{1}(\Omega)$ and $H_{0}\left(\partial_{3}, \Omega\right)$ :

$$
\begin{gathered}
H_{b}^{2}(\Omega)={\overline{C_{b}^{\infty}(\Omega)}}^{H^{2}(\Omega)}=\left\{v \in H^{2}(\Omega) ; v=\frac{\partial v}{\partial n}=0 \text { on } \Gamma_{b}\right\}, \\
\left.H^{1}\left(\partial_{3}, \Omega\right)=\left\{v \in H^{1}(\Omega) ; \partial_{3} v \in H^{1} \Omega\right)\right\}, \\
H_{0}^{1}\left(\partial_{3}, \Omega\right)={\overline{C_{0}^{\infty}(\Omega)}}^{1}\left(\partial_{3}, \Omega\right) \\
=\left\{v \in H^{1}\left(\partial_{3}, \Omega\right) ; v=\partial_{3} v=0 \text { on } \partial \Omega\right\} .
\end{gathered}
$$

Indeed, as $v_{H} \in Y_{H}$, there exists a sequence $\left(\varphi_{H}^{n}, \varphi_{3}^{n}\right) \in \mathcal{U}$ such that $\varphi_{H}^{n} \rightarrow v_{H}$ in $H^{2}(\Omega)^{2}$. Then $\partial_{3} \varphi_{3}^{n}=-\partial_{1} \varphi_{1}^{n}-\partial_{2} \varphi_{2}^{n}$ is a Cauchy sequence in $H^{1}(\Omega)$, and by vertical Poincaré inequality, $\varphi_{3}^{n}$ is also a Cauchy sequence in $H^{1}(\Omega)$. Therefore, $\varphi_{3}^{n}$, being a Cauchy sequence in $H^{1}\left(\partial_{3}, \Omega\right)$, converges to a function $v_{3}$, which provides the desired lifting function. The continuous dependence (6.3) results from the above construction.

Now we take this $\mathbf{v}=\left(v_{H}, v_{3}\right)$ as a test function in (6.2) and integrate over $(t, t+h)$; i.e.,

$$
\left(\tau_{h} u_{H}(t)-u_{H}(t), v_{H}\right)+\epsilon^{2}\left(\tau_{h} u_{3}(t)-u_{3}(t), v_{3}\right)=\int_{t}^{t+h} g^{\epsilon}(s) \mathrm{ds}
$$

where

$$
\begin{aligned}
g^{\epsilon}(s)= & \left(u_{H},(\mathbf{u} \cdot \nabla) v_{H}\right)-\epsilon^{2}\left(\mathbf{u} \cdot \nabla u_{3}, v_{3}\right)-\left(b\left(u_{H}\right), v_{H}\right)-\left(\nabla_{\nu} u_{H}, \nabla_{\nu} v_{H}\right) \\
& -\epsilon\left(\nabla_{\nu}\left(\epsilon u_{3}\right), \nabla_{\nu} v_{3}\right)-\epsilon\left\{\left(\beta u_{3}, v_{1}\right)-\left(\beta u_{1}, v_{3}\right)\right\}+\left\langle\theta_{H}, v_{H}\right\rangle_{\Gamma_{s}} .
\end{aligned}
$$

Now we prove that

$$
\left\|g^{\epsilon}\right\|_{L^{4 / 3}(0, T)} \leq C\left\|v_{H}\right\|_{Y_{H}} .
$$

To this end, we estimate every piece of $g^{\epsilon}$. For the nonlinear terms, we have

$$
\left(u_{H},(\mathbf{u} \cdot \nabla) v_{H}\right) \leq\left\|u_{H}\right\|_{L^{3}}\|\mathbf{u}\|_{L^{2}}\left\|\nabla v_{H}\right\|_{L^{6}} \leq C\left\|u_{H}\right\|_{L^{3}}\|\mathbf{u}\|_{L^{2}}\left\|v_{H}\right\|_{Y_{H}}
$$

and

$$
\epsilon^{2}\left(\mathbf{u} \cdot \nabla u_{3}, v_{3}\right) \leq\|\epsilon \mathbf{u}\|_{L^{3}}\left\|\nabla\left(\epsilon u_{3}\right)\right\|_{L^{2}}\left\|v_{3}\right\|_{L^{6}} \leq C\|\epsilon \mathbf{u}\|_{L^{3}}\left\|\epsilon u_{3}\right\|_{H^{1}}\left\|v_{H}\right\|_{Y_{H}} .
$$

By interpolation between $L^{\infty}\left(0, T ; L^{2}\right)$ and $L^{2}\left(0, T ; L^{6}\right), u_{H}$ is bounded in $L^{4}\left(0, T ; L^{3}\right)$; i.e., $\left\|u_{H}\right\|_{L^{3}}$ is bounded in $L^{4}(0, T)$. As $\|\mathbf{u}\|_{L^{2}}$ is bounded in $L^{2}(0, T)$, we have $\left(u_{H},(\mathbf{u} \cdot \nabla) v_{H}\right)$ bounded in $L^{4 / 3}(0, T)$. Similarly, as $\|\epsilon \mathbf{u}\|_{L^{3}}$ is bounded in $L^{4}(0, T)$ and $\left\|\epsilon u_{3}\right\|_{H^{1}}$ is bounded in $L^{2}(0, T)$, we have $\epsilon^{2}\left(\mathbf{u} \cdot \nabla u_{3}, v_{3}\right)$ bounded in $L^{4 / 3}(0, T)$. The linear terms of $g^{\epsilon}$ are handled easily by the Cauchy-Schwarz inequality:

$$
\begin{aligned}
\left(b\left(u_{H}\right), v_{H}\right) & \leq\left\|u_{H}\right\|_{L^{2}}\left\|v_{H}\right\|_{L^{2}} & & \text { bounded in } L^{\infty}(0, T), \\
\left(\nabla_{\nu} u_{H}, \nabla_{\nu} v_{H}\right) & \leq\left\|u_{H}\right\|_{H^{1}}\left\|v_{H}\right\|_{H^{1}} & & \text { bounded in } L^{2}(0, T), \\
\epsilon\left(\nabla_{\nu}\left(\epsilon u_{3}\right), \nabla_{\nu} v_{3}\right) & \leq \epsilon\left\|\epsilon u_{3}\right\|_{H^{1}}\left\|v_{3}\right\|_{H^{1}} & & \text { bounded in } L^{2}(0, T), \\
\epsilon \beta\left\{\left(u_{3}, v_{1}\right)-\left(u_{1}, v_{3}\right)\right\} & \leq 2 f\|\epsilon \mathbf{u}\|_{L^{2}}\|\mathbf{v}\|_{L^{2}} & & \text { bounded in } L^{\infty}(0, T), \\
\left\langle\theta_{H}, v_{H}\right\rangle_{\Gamma_{s}} & \leq C\left\|\theta_{H}\right\|_{H^{-1 / 2}\left(\Gamma_{s}\right)}\left\|v_{H}\right\|_{H^{1}} & & \text { bounded in } L^{2}(0, T) .
\end{aligned}
$$


Therefore, taking into account (6.3), according to all previous bounds, (6.5) holds. Next, applying the Hölder inequality to (6.5), we see that

$$
\int_{t}^{t+h}\left|g^{\epsilon}(s)\right| \mathrm{ds} \leq C h^{1 / 4}\left\|v_{H}\right\|_{Y_{H}}
$$

On the other hand,

$$
\left|\epsilon^{2}\left(\tau_{h} u_{3}(t)-u_{3}(t), v_{3}\right)\right| \leq \epsilon\left\{\left\|\tau_{h}\left(\epsilon u_{3}\right)(t)\right\|_{L^{2}}+\left\|\epsilon u_{3}(t)\right\|_{L^{2}}\right\}\left\|v_{3}\right\|_{L^{2}} \leq \epsilon C\left\|v_{H}\right\|_{Y_{H}}
$$

by virtue of Proposition 6.1 .

These last two estimates together with (6.4) yield the required result.

6.3. Convergence. Here we come back to the notation $\mathbf{u}^{\epsilon}$. The space-time weak form of the anisotropic Navier-Stokes equations (2.7)-(2.13) is as follows.

Find $\mathbf{u}^{\epsilon}=\left(u_{H}^{\epsilon}, u_{3}^{\epsilon}\right) \in L^{2}(0, T ; \mathbf{V}) \cap L^{\infty}\left(0, T ; L^{2}(\Omega)^{3}\right)$ such that

$$
\begin{aligned}
& \int_{0}^{T}-\left(u_{H}^{\epsilon}, \partial_{t} v_{H}\right)-\left(u_{H}^{\epsilon},\left(\mathbf{u}^{\epsilon} \cdot \nabla\right) v_{H}\right)+\left(b\left(u_{H}^{\epsilon}\right), v_{H}\right)+\left(\nabla_{\nu} u_{H}^{\epsilon}, \nabla_{\nu} v_{H}\right) \\
& (6.6)+\epsilon^{2} \int_{0}^{T}-\left(u_{3}^{\epsilon}, \partial_{t} v_{3}\right)+\left(\mathbf{u}^{\epsilon} \cdot \nabla u_{3}^{\epsilon}, v_{3}\right)+\left(\nabla_{\nu} u_{3}^{\epsilon}, \nabla_{\nu} v_{3}\right) \\
& \quad+\epsilon \beta \int_{0}^{T}\left(u_{3}^{\epsilon}, v_{1}\right)-\left(u_{1}^{\epsilon}, v_{3}\right)=-\left(u_{0 H}, v_{H}(0)\right)-\epsilon^{2}\left(u_{03}, v_{3}(0)\right)+\int_{0}^{T}\left\langle\theta_{H}, v_{H}\right\rangle_{\Gamma_{s}} \\
& \quad \forall \mathbf{v}=\left(v_{H}, v_{3}\right) \in H^{1}(0, T ; \mathbf{V}), \operatorname{with} \mathbf{v}(T)=0 .
\end{aligned}
$$

The purpose of the following is to take the limit as $\epsilon \rightarrow 0$ in (6.6) to come to (4.1). By Propositions 6.1 and 6.2 , it follows that $\mathbf{u}^{\epsilon}$ is bounded in $L^{2}(0, T ; \mathbf{W})$ and $u_{H}^{\epsilon}$ is bounded in $L^{\infty}\left(0, T ; B_{H}\right)$, allowing us to extract a subsequence, still denoted by $\mathbf{u}^{\epsilon}$, such that

$$
\begin{gathered}
\mathbf{u}^{\epsilon}=\left(u_{H}^{\epsilon}, u_{3}^{\epsilon}\right) \rightarrow \mathbf{u}=\left(u_{H}, u_{3}\right) \quad \text { in } L^{2}(0, T ; \mathbf{W}) \text { weak }, \\
u_{H}^{\epsilon} \stackrel{\star}{\lessgtr} u_{H} \quad \text { in } L^{\infty}\left(0, T ; B_{H}\right) \text { weak }-\star
\end{gathered}
$$

These weak convergences are enough to take the limit in the linear terms of (6.6) (cf. [1]). In particular, the terms of $\mathrm{O}(\epsilon)$ associated with the Coriolis acceleration vanish as $\epsilon$ tends to zero. Indeed,

$$
\begin{gathered}
\epsilon \beta \int_{0}^{T}\left(u_{3}^{\epsilon}, v_{1}\right)-\left(u_{1}^{\epsilon}, v_{3}\right) \leq \epsilon 2 f \int_{0}^{T}\left\|\mathbf{u}^{\epsilon}\right\|_{L^{2}}\|\mathbf{v}\|_{L^{2}} \\
\leq \epsilon 2 f\left\|\mathbf{u}^{\epsilon}\right\|_{L^{2}\left(0, T ; L^{2}\right)}\|\mathbf{v}\|_{L^{2}\left(0, T ; L^{2}\right)} \leq \epsilon C\|\mathbf{v}\|_{L^{2}\left(0, T ; L^{2}\right)} \leq C \epsilon .
\end{gathered}
$$

On the other hand, combining (6.1), Proposition 6.1, and Lemma 6.3, we can apply Theorem 5.1 for $p=\infty$ and the spaces $B_{H} \stackrel{\text { compact }}{\hookrightarrow} W_{H}^{\prime} \hookrightarrow Y_{H}^{\prime}$. Therefore, there exists a subsequence $u_{H}^{\epsilon} \rightarrow u_{H}$ in $\mathcal{C}\left(0, T ; W_{H}^{\prime}\right)$ strong. Thus we get the weak time-continuity $u_{H} \in \mathcal{C}\left(0, T ; W_{H}^{\prime}\right)$, so that the initial condition $(2.20)$ makes sense for the horizontal velocities. On the other hand, the term of $0\left(\epsilon^{2}\right)$ related to the initial condition for the vertical velocity vanishes as $\epsilon$ tends to zero. Indeed,

$$
-\epsilon^{2}\left(u_{03}, v_{3}(0)\right) \leq \epsilon^{2}\left\|u_{03}\right\|_{L^{2}}\left\|v_{3}(0)\right\|_{L^{2}} \leq \epsilon^{2}\left\|\mathbf{u}_{0}\right\|_{L^{2}}\left\|v_{3}\right\|_{\mathcal{C}\left(0, T ; L^{2}\right)} \leq C \epsilon^{2} .
$$


Now the nonlinear terms fall into four types:

$$
\begin{aligned}
& \text { (I) } \epsilon^{2} \int_{0}^{T}\left(u_{i}^{\epsilon} \partial_{i} u_{3}^{\epsilon}, v_{3}\right) \mathrm{dt}, \quad 1 \leq i \leq 2, \\
& \text { (II) } \epsilon^{2} \int_{0}^{T}\left(u_{3}^{\epsilon} \partial_{3} u_{3}^{\epsilon}, v_{3}\right) \mathrm{dt}, \\
& \text { (III) } \int_{0}^{T}\left(u_{i}^{\epsilon} u_{j}^{\epsilon}, \partial_{j} v_{i}\right) \mathrm{dt}, \quad 1 \leq i, j \leq 2, \\
& \text { (IV) } \quad \int_{0}^{T}\left(u_{i}^{\epsilon} u_{3}^{\epsilon}, \partial_{3} v_{i}\right) \mathrm{dt}, \quad 1 \leq i \leq 2 .
\end{aligned}
$$

Type (I) term:

$$
\begin{gathered}
\epsilon^{2} \int_{0}^{T}\left(u_{i}^{\epsilon} \partial_{i} u_{3}^{\epsilon}, v_{3}\right) \mathrm{dt} \leq \epsilon \int_{0}^{T}\left\|u_{i}^{\epsilon}\right\|_{L^{6}}\left\|\partial_{i}\left(\epsilon u_{3}^{\epsilon}\right)\right\|_{L^{2}}\left\|v_{3}\right\|_{L^{3}} \\
\leq C \epsilon\left\|u_{i}^{\epsilon}\right\|_{L^{2}\left(0, T ; H^{1}\right)}\left\|\partial_{i}\left(\epsilon u_{3}^{\epsilon}\right)\right\|_{L^{2}\left(0, T ; L^{2}\right)}\left\|v_{3}\right\|_{\mathcal{C}\left(0, T ; H^{1}\right)} \leq C \epsilon .
\end{gathered}
$$

Type (II) term:

$$
\begin{gathered}
\epsilon^{2} \int_{0}^{T}\left(u_{3}^{\epsilon} \partial_{3} u_{3}^{\epsilon}, v_{3}\right) \mathrm{dt} \leq \epsilon \int_{0}^{T}\left\|\epsilon u_{3}^{\epsilon}\right\|_{L^{6}}\left\|\partial_{3} u_{3}^{\epsilon}\right\|_{L^{2}}\left\|v_{3}\right\|_{L^{3}} \\
\leq C \epsilon\left\|\epsilon u_{3}^{\epsilon}\right\|_{L^{2}\left(0, T ; H^{1}\right)}\left\|\partial_{3} u_{3}^{\epsilon}\right\|_{L^{2}\left(0, T ; L^{2}\right)}\left\|v_{3}\right\|_{\mathcal{C}\left(0, T ; H^{1}\right)} \leq C \epsilon .
\end{gathered}
$$

Consequently, the type (I) and (II) terms are $O(\epsilon)$ and vanish as $\epsilon$ tends to zero.

To handle the type (III) and (IV) terms, we need some strong convergences. From compactness by perturbation (Theorem 5.1 for $p=2$ and the spaces $W_{H} \stackrel{\text { compact }}{\hookrightarrow}$ $\left.B_{H} \hookrightarrow Y_{H}^{\prime}\right)$, there exists a subsequence, still denoted by $u_{H}^{\epsilon}$, such that

$$
u_{H}^{\epsilon} \rightarrow u_{H} \quad \text { in } L^{2}\left(0, T ; L^{2}(\Omega)^{2}\right) \equiv L^{2}((0, T) \times \Omega)^{2} \text { strong. }
$$

By Proposition 6.1, we have $u_{H}^{\epsilon}$ bounded in $L^{\infty}\left(0, T ; L^{2}(\Omega)^{2}\right) \cap L^{2}\left(0, T ; L^{6}(\Omega)^{2}\right)$, which by interpolation ensures that

$$
u_{H}^{\epsilon} \text { is bounded in } L^{10 / 3}\left(0, T ; L^{10 / 3}(\Omega)^{2}\right) \equiv L^{10 / 3}((0, T) \times \Omega)^{2} .
$$

By the interpolation inequality again, for all $q: 2 \leq q<10 / 3$ there exists $\alpha: 0<\alpha \leq$ 1 such that

$$
\left\|u_{H}^{\epsilon}-u_{H}\right\|_{L^{q}} \leq\left\|u_{H}^{\epsilon}-u_{H}\right\|_{L^{2}}^{\alpha}\left\|u_{H}^{\epsilon}-u_{H}\right\|_{L^{10 / 3}}^{1-\alpha}
$$

Thus

$$
u_{H}^{\epsilon} \rightarrow u_{H} \quad \text { in } L^{q}((0, T) \times \Omega) \text { strong } \quad \forall q: 2 \leq q<10 / 3 .
$$

Type (III) term: By the Hölder inequality and (6.8), we have

$$
u_{i}^{\epsilon} u_{j}^{\epsilon} \rightarrow u_{i} u_{j} \quad \text { in } L^{r}((0, T) \times \Omega) \text { strong } \quad \forall r: 1 \leq r<5 / 3
$$


and for all $i, j=1,2$. On the other hand, by interpolation between $L^{\infty}\left(0, T ; L^{2}\right)$ and $L^{2}\left(0, T ; L^{6}\right), u_{i}^{\epsilon}$ (and $\left.u_{j}^{\epsilon}\right)$ is bounded in $L^{8 / 3}\left(0, T ; L^{4}\right)$, and hence $u_{i}^{\epsilon} u_{j}^{\epsilon}$ is bounded in $L^{4 / 3}\left(0, T ; L^{2}\right)$, and finally,

$$
u_{i}^{\epsilon} u_{j}^{\epsilon} \rightarrow u_{i} u_{j} \quad \text { in } L^{4 / 3}\left(0, T ; L^{2}\right) \text { weak, } \quad 1 \leq i, \quad j \leq 2 .
$$

In particular, we get

$$
\int_{0}^{T}\left(u_{i}^{\epsilon} u_{j}^{\epsilon}, \partial_{j} v_{i}\right) \rightarrow \int_{0}^{T}\left(u_{i} u_{j}, \partial_{j} v_{i}\right), \quad 1 \leq i, \quad j \leq 2 .
$$

Indeed, $v_{i} \in \mathcal{C}\left(0, T ; H^{1}\right)$ so that

$$
\partial_{j} v_{i} \in L^{\infty}\left(0, T, L^{2}\right) \subset L^{4}\left(0, T, L^{2}\right) \equiv\left(L^{4 / 3}\left(0, T, L^{2}\right)\right)^{\prime} .
$$

Type (IV) term: We have

$$
u_{3}^{\epsilon} \rightarrow u_{3} \quad \text { in } L^{2}\left(0, T ; L^{2}\right) \text { weak. }
$$

So by the Hölder inequality and (6.8),

$$
u_{i}^{\epsilon} u_{3}^{\epsilon} \longrightarrow u_{i} u_{3} \quad \text { in } L^{s}\left(0, T ; L^{s}\right) \text { weak } \quad \forall s: 1 \leq s<5 / 4
$$

and for all $i=1,2$. On the other hand, it is easy to see that $u_{i}^{\epsilon} u_{3}^{\epsilon}$ is bounded in $L^{8 / 7}\left(0, T ; L^{4 / 3}\right)$, and hence

$$
u_{i}^{\epsilon} u_{3}^{\epsilon} \rightarrow u_{i} u_{3} \quad \text { in } L^{8 / 7}\left(0, T ; L^{4 / 3}\right) \text { weak, } \quad 1 \leq i \leq 2 .
$$

Now we shall have to slightly increase the regularity of the test functions of (4.1) to finish the limit process in the Type (IV) terms. For instance, assuming the additional regularity for the test functions $\partial_{3} v_{i} \in L^{8}\left(0, T ; L^{4}\right)$, we get

$$
\int_{0}^{T}\left(u_{i}^{\epsilon} u_{3}^{\epsilon}, \partial_{3} v_{i}\right) \rightarrow \int_{0}^{T}\left(u_{i} u_{3}, \partial_{3} v_{i}\right), \quad 1 \leq i \leq 2
$$

In conclusion, the limit function $\mathbf{u}$ is a solution of the variational formulation (4.1) for all $\mathbf{v}=\left(v_{H}, v_{3}\right) \in H^{1}(0, T, \mathbf{V})$ with $v_{H}(T)=0$ and $\partial_{3} v_{H} \in L^{8}\left(0, T ; L^{4}\right)$. Finally, we can argue by density, taking advantage of the regularity of each term of (4.1), and obtain that (4.1) holds for all $\mathbf{v}=\left(v_{H}, v_{3}\right) \in H^{1}(0, T, \mathbf{W})$ with $v_{H}(T)=0$ and $\partial_{3} v_{H} \in L^{\infty}\left(0, T ; L^{3}\right)$; hence the proof of Theorem 3.1 is finished.

\section{Concluding remarks.}

7.1. Convergence of the pressure. By using the De Rham lemma [18] in the formulation (6.6) (resp., (4.1)), we can recover the potentials $p^{\epsilon}$ (resp., $p$ ) as distributions

$$
\nabla p^{\epsilon}=\left(\begin{array}{l}
-\partial_{t} u_{1}^{\epsilon}-\mathbf{u}^{\epsilon} \cdot \nabla u_{1}^{\epsilon}+\Delta_{\nu} u_{1}^{\epsilon}+\alpha u_{2}^{\epsilon}-\epsilon \beta u_{3}^{\epsilon} \\
-\partial_{t} u_{2}^{\epsilon}-\mathbf{u}^{\epsilon} \cdot \nabla u_{2}^{\epsilon}+\Delta_{\nu} u_{2}^{\epsilon}-\alpha u_{1}^{\epsilon} \\
-\epsilon^{2}\left\{\partial_{t} u_{3}^{\epsilon}+\mathbf{u}^{\epsilon} \cdot \nabla u_{3}^{\epsilon}-\Delta_{\nu} u_{3}^{\epsilon}\right\}+\epsilon \beta u_{1}^{\epsilon}
\end{array}\right)
$$

respectively,

$$
\nabla p=\left(\begin{array}{c}
-\partial_{t} u_{1}-\mathbf{u} \cdot \nabla u_{1}+\Delta_{\nu} u_{1}+\alpha u_{2} \\
-\partial_{t} u_{2}-\mathbf{u} \cdot \nabla u_{2}+\Delta_{\nu} u_{2}-\alpha u_{1} \\
0
\end{array}\right)
$$


Moreover, (7.1) is also verified in $H^{-1}\left(0, T ; H^{-1}(\Omega)^{3}\right)$ (i.e., in the dual space of $\left.H_{0}^{1}\left(0, T ; H_{0}^{1}(\Omega)^{3}\right)\right)$, whereas $(7.2)$ holds in $H^{-1}\left(0, T ; W^{-1,3 / 2}(\Omega)^{3}\right)$ (i.e., in the dual space of $\left.H_{0}^{1}\left(0, T ; W_{0}^{1,3}(\Omega)^{3}\right)\right)$. Proceeding as in subsection 6.3 , we may derive that

$$
\partial_{i} p^{\epsilon} \stackrel{\star}{\rightarrow} \partial_{i} p \quad \text { in } H^{-1}\left(0, T ; W^{-1,3 / 2}(\Omega)\right), \quad i=1,2,
$$

and

$$
\left\|\partial_{3} p^{\epsilon}\right\|_{H^{-1}\left(0, T ; H^{-1}(\Omega)\right)} \leq C \epsilon .
$$

In particular, we have the strong convergence of $\partial_{3} p^{\epsilon}$ to $\partial_{3} p$.

Remark. The strong convergence of $\partial_{3} p^{\epsilon}$ takes place in a better space than the weak convergence of $\partial_{i} p^{\epsilon}, i=1,2$. In some sense, this means that the validity of the hydrostatic approximation is less demanding than the validity of the horizontal momentum equations.

Remark. The above convergences can be slightly improved with respect to time. They remain true, replacing the space $H^{-1}\left(0, T ; W^{-1,3 / 2}(\Omega)\right)$ (resp., $H^{-1}\left(0, T ; H^{-1}(\Omega)\right)$ ) with the space $W^{-1, \infty}\left(0, T ; W^{-1,3 / 2}(\Omega)\right)$ (resp., $W^{-1, \infty}\left(0, T ; H^{-1}(\Omega)\right)$ ).

7.2. Orders of magnitude of the vertical velocity in the original domain. The purpose of this last subsection is to interpret the previous results in the original domain $\Omega_{\epsilon}$. Consequently, we are going to consider $\mathbf{v}=\left(v_{1}, v_{2}, v_{3}\right)$, the weak solution in $\Omega_{\epsilon}$ of problem (2.1)-(2.5), related to $\mathbf{u}^{\epsilon}=\left(u_{1}^{\epsilon}, u_{2}^{\epsilon}, u_{3}^{\epsilon}\right)$, a weak solution of problem $(2.7)-(2.13)$ in $\Omega$; see (2.6). First, it is important to notice that the true vertical velocity $v_{3}=\epsilon u_{3}^{\epsilon}$ is small with respect to the horizontal velocities $v_{i}, i=1,2$. Indeed, taking into account the estimates in Proposition 6.1 for $u_{i}^{\epsilon}, i=1,2$, and Proposition 6.2 for $u_{3}^{\epsilon}$, scaling off $\Omega$ to $\Omega_{\epsilon}$, we obtain

$$
\frac{\left\|v_{3}\right\|_{L^{2}\left(0, T ; L^{2}\left(\Omega_{\epsilon}\right)\right)}}{\left\|v_{i}\right\|_{L^{2}\left(0, T ; L^{2}\left(\Omega_{\epsilon}\right)\right)}}=0(\epsilon), \quad i=1,2 .
$$

By the same argument, we obtain

$$
\frac{\left\|\partial_{z} v_{3}\right\|_{L^{2}\left(0, T ; L^{2}\left(\Omega_{\epsilon}\right)\right)}}{\left\|\partial_{z} v_{i}\right\|_{L^{2}\left(0, T ; L^{2}\left(\Omega_{\epsilon}\right)\right)}}=0(\epsilon), \quad i=1,2 .
$$

This phenomenon is actually observed in most geophysical flows, which, therefore, are quasi-horizontal. It is striking that the vertical velocity goes to zero even if the initial vertical velocity is not assumed to be small. Looking at (6.7) in the proof of convergence, we need only that $\epsilon^{2}\left\|u_{03}\right\|_{L^{2}(\Omega)} \rightarrow 0$, that is, $\left\|v_{03}\right\|_{L^{2}\left(\Omega_{\epsilon}\right)} /\left\|v_{0 i}\right\|_{L^{2}\left(\Omega_{\epsilon}\right)}=$ $O\left(\epsilon^{-\alpha}\right), \alpha<1$.

Whereas, for the horizontal gradient, we cannot avail ourselves of Proposition 6.2, and we obtain only

$$
\frac{\left\|\nabla_{x, y} v_{3}\right\|_{L^{2}\left(0, T ; L^{2}\left(\Omega_{\epsilon}\right)\right)}}{\left\|\nabla_{x, y} v_{i}\right\|_{L^{2}\left(0, T ; L^{2}\left(\Omega_{\epsilon}\right)\right)}}=0(1), \quad i=1,2 .
$$

\section{REFERENCES}

[1] P. AzÉRAD, Analyse des équations de Navier-Stokes en bassin peu profond et de l'équation de transport, Thèse de Doctorat ès sciences, Université de Neuchâtel, Neuchâtel, Switzerland, 1996; also available online from http://gala.univ-perp.fr/ ${ }^{\sim}$ azerad. 
[2] P. AzÉrad ANd F. Gulllén, Equations de Navier-Stokes en bassin peu profond: hydrostatique l'approximation, C. R. Acad. Sci. Paris Sér. I Math., 329 (1999), pp. 961-966.

[3] P. Azérad, O. Besson, And F. Guillén, Fluid flow in shallow domains: Mathematical analysis and numerical simulation, in Proceedings of the IV Catalan Days of Applied Mathematics, C. Garcia, C. Olivé, and M. Sanroma, eds., Universitat Rovira i Virgili, Tarragona, Spain, 1998, pp. 9-16.

[4] O. Besson, M. R. Laydi, And R. Touzani, Un modèle asymptotique en océanographie, C. R. Acad. Sci. Paris Sér. I Math., 310 (1990), pp. 661-665.

[5] O. Besson And M. R. LAYdi, Some estimates for the anisotropic Navier-Stokes equations and for the hydrostatic approximation, M2AN Math. Model. Numer. Anal., 26 (1992), pp. $855-865$.

[6] D. Bresch, J. Lemoine, And J. Simon, A vertical diffusion model for lakes, SiAM J. Math. Anal., 30 (1999), pp. 603-622.

[7] D. Iftimie, The 3D Navier-Stokes equations seen as a perturbation of the $2 D$ Navier-Stokes equations, Bull. Soc. Math. France, 127 (1999), pp. 473-517.

[8] O. A. Ladyzhenskaya, The Mathematical Theory of Viscous Incompressible Flow, Gordon and Breach, New York, 1969.

[9] R. Lewandowski, Analyse Mathématique et Océanographie, Masson, Paris, 1997.

[10] J.-L. Lions, Quelques méthodes de résolution des problèmes aux limites non linéaires, Dunod, Paris, 1969.

[11] J.-L. Lions, R. Temam, And S. Wang, New formulations of the primitive equations of the atmosphere and applications, Nonlinearity, 5 (1992), pp. 237-288.

[12] J.-L. Lions, R. Temam, And S. Wang, On the equations of large scale ocean, Nonlinearity, 5 (1992), pp. 1007-1053.

[13] S. Montgomery-Smith, Global regularity of the Navier Stokes equation on thin threedimensional domains with periodic boundary conditions, Electron. J. Differential Equations, 1999 (1999), pp. 1-19; also available online from http://ejde.math.unt.edu.

[14] B. Pascal, De l'équilibre des liqueurs, Paris, 1663, in Oeuvres complètes, coll. La Pléiade, Gallimard, Paris, 1998.

[15] J. Pedlosky, Geophysical Fluid Dynamics, Springer-Verlag, New York, 1987.

[16] G. Raugel And G. Sell, Navier-Stokes equations in thin 3D domains I: Global attractors and global regularity of solutions, J. Amer. Math. Soc., 6 (1993) pp. 503-568.

[17] J. Simon, Compact sets in $L^{p}(0, T ; B)$, Ann. Mat. Pura Appl. (4), 146 (1987), pp. 65-97.

[18] R. Temam, Navier-Stokes Equations, North-Holland, Elsevier, Amsterdam, 1985.

[19] R. Temam, Sur la stabilité et la convergence de la méthode des pas fractionnaires, Ann. Mat. Pura Appl. (4), 79 (1968), pp. 191-379.

[20] R. Temam And M. Ziane, Navier-Stokes equations in three-dimensional thin domains with various boundary conditions, Adv. Differential Equations, 1 (1996), pp. 499-546.

[21] R. Temam And M. Ziane, Navier-Stokes equations in thin spherical domains, in Optimization Methods in Partial Differential Equations, Contemp. Math. 209, AMS, Providence, RI, 1997, pp. 281-314.

[22] R. K. Zeytounian, Modélisation asymptotique en mécanique des fluides newtoniens, SpringerVerlag, New York, 1994. 